\title{
Effects of Cognitive Impairment on vowel duration
}

Charalambos Themistocleous ${ }^{1,2}$, Dimitrios Kokkinakis ${ }^{1}$, Marie Eckerström $^{3}$, Kathleen Fraser ${ }^{1}$, Kristina Lundholm Fors ${ }^{1}$

${ }^{1}$ Department of Swedish, University of Gothenburg, Sweden

2Department of Neurology, Johns Hopkins University, USA

${ }^{3}$ Department of Psychiatry and Neurochemistry, University of Gothenburg, Sweden

https://doi.org/10.36505/ExLing-2018/09/0027/000360

\begin{abstract}
Mild cognitive impairment (MCI) is a neurological condition, which is characterized by a noticeable decline of cognitive abilities, including communicative and linguistic skills. In this study, we have measured the duration of vowels produced in a reading task by 55 speakers - 30 healthy controls and $25 \mathrm{MCI}-$. The main results showed that MCI speakers differed significantly from HC in vowel duration as MCI speakers produced overall longer vowels. Also, we found that gender effects on vowel duration were different in MCI and HC. One significant aspect of this finding is that they highlight the contribution of vowel acoustic features as markers of MCI.

Key words: MCI/AD, vowel duration, language pathologies, Swedish
\end{abstract}

\section{Introduction}

Mild Cognitive Impairment is a neurodegenerative condition that causes small but noticeable and measurable decline in cognitive abilities, including memory and language skills that differ from normal ageing. Often MCI is accompanied by depression, anxiety, aggression, irritability, and apathy. People with MCI have memory difficulties-such as remembering events and situations-in decision making, planning, interpreting instructions, and in finding their way in familiar environments. There is not a single cause of MCI, thought biomedicalsuch as shrinkage of the hippocampus, enlargement of the ventricles, abnormal clumps of beta-amyloid protein and tau-genetic-such as the presence of the APOE-e4 gene also linked with AD-and lifestyle factors-such as smoking, high blood pressure, luck of physical exercise, and diabetes, are high risk factors that can contribute to MCI. The aim of this is study is to determine the effects of MCI vs healthy controls (HC) on vowel duration. Vowel duration is extremely sensitive to linguistic (such as vowel quality, stress, positive in the utterance, etc.), emotional, and physiological (age, medical condition etc.) factors (Haley \& Overton, 2001, Themistocleous 2017). Previous research showed that vowel duration can be affected in MCI (Jarrold, et al. 2014).

ExLing 2018: Proceedings of 9th Tutorial and Research Workshop on Experimental Linguistics, 28-30 August, Paris, Frannce 
114 Ch. Themistocleous et al.

\section{Methodology}

The acoustic materials of this study were collected from a subcohort of the Gothenburg MCI study (Wallin et al., 2016), which is a large longitudinal research on MCI. Specifically, 13 female and 12 male participants with MCI and 19 female and 11 male healthy control (HC) speakers participated in this study. The two groups did not differ with respect to age $(t(52.72)=-1.8178, p=$ n.s. $)$ and gender $(\mathrm{W}=1567.5, \mathrm{p}$ $=$ n.s.), as it is evident by the non-significant results from a $t$ test and an independent 2-group Mann-Whitney U Test respectively. All speakers were native speakers of Swedish. Speaker selection was based on certain inclusion and exclusion criteria: they should not suffer from dyslexia, deep depression, substance abuse, and other serious psychiatric, neurological or brain-related diseases, such as Parkinson's disease. Ethic approvals for the study were obtained by the local ethical committee review board (number: L09199, 1999; T479- 11, 2011); while the currently described study was approved by the local ethical committee decision 206-16, 2016 and T021-18.

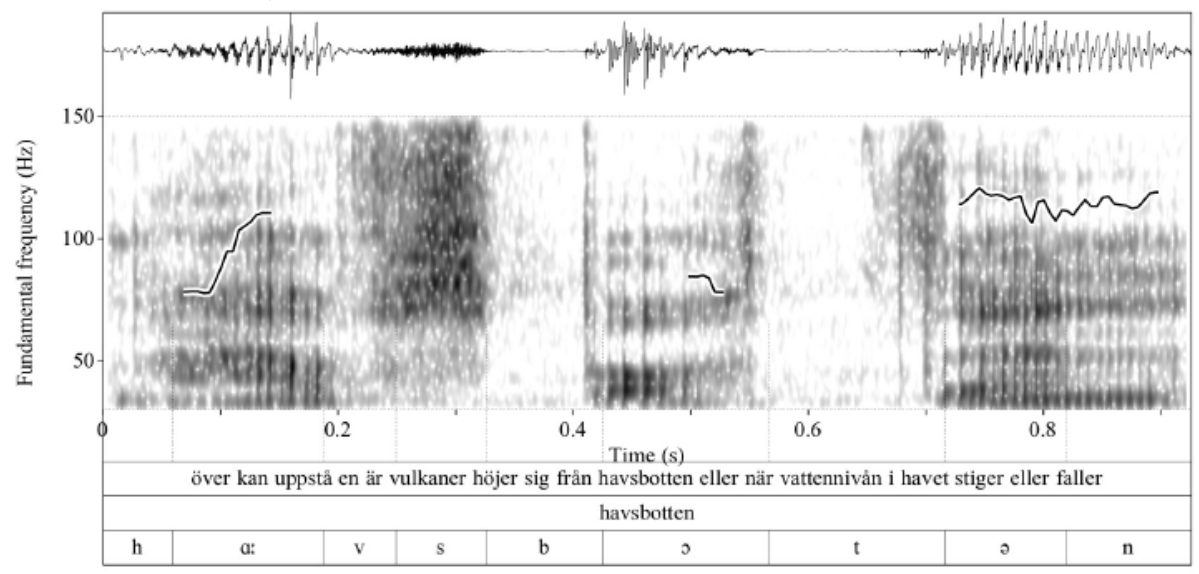

Figure 1. An example of one transcription generated using Themis-SV.

The data were collected from a reading task where participants read a short passage. Participants were instructed to read the text aloud and without interruptions. The narratives were audio-recorded and transcribed and segmented using Themis-SV a system for the automatic transcription of Swedish. The system processes these recordings and returns an output with three tiers: the utterance tier, the word tier, and the vowels and consonants tier (see an example output Figure 1). The output of the system is a fast and reliable transcription and segmentation of speech, which is very close to transcriptions and segmentations 
performed manually. The automatic segmentation of speech enables targeted acoustic measurements, such as measurements of consonant spectra, formant frequencies of vowels, fundamental frequency, pauses, speech rate, etc. and other acoustic measurements that have been known to differentiate between the different types of language disorders. All automatic transcriptions were evaluated manually by the first author. For the statistical analysis, we run a linear mixed effects model:

duration $\sim$ condition $+(1 \mid$ gender $)+(1 \mid$ vowel $)$

where duration is a depended variable, condition (MCI vs. HC) a fixed factor, and gender and vowel are random intercepts. The duration was log-transformed to correct for normality. Linear mixed model was fit by REML t-tests using Satterthwaite approximations to degrees of freedom. All statistics were conducted in R using the lme 4 and lmerTest packages.

\section{Results}

The mean values and the standard deviation (SD) are presented in Table 1. The results of the statistical model are presented in Table 2. Overall, MCI speakers produced longer vowels whereas HC produced shorter vowels. This finding was significant (see Table 2).

Table 1. Mean and SD of vowel duration in ms for HC and MCI female and male participants.

\begin{tabular}{lllll}
\hline \hline & HC & & MCI & \\
\hline & Mean & SD & Mean & SD \\
\hline Female & 99 & 56 & 101 & 60 \\
\hline Male & 89 & 52 & 98 & 58 \\
\hline \hline
\end{tabular}

Table 2. Results of the linear mixed effects model for the effects of condition on the log-transformed duration.

\begin{tabular}{llllll}
\hline \hline & Estimate & $\mathrm{SE}$ & $\mathrm{df}$ & $\mathrm{t}$ value & $\operatorname{Pr}(>|\mathrm{t}|)$ \\
\hline Intercept & 4.528 & 0.082 & 9 & 55.154 & .0001 \\
\hline Condition MCI & 0.043 & 0.009 & 13070 & 4.902 & .0001 \\
\hline \hline
\end{tabular}

Table 3. Results of the linear mixed effects model for the effects of the interaction condition $\times$ gender on the log-transformed duration.

\begin{tabular}{llllll}
\hline \hline & Estimate & $\mathrm{SE}$ & $\mathrm{df}$ & $\mathrm{t}$ value & $\operatorname{Pr}(>|\mathrm{t}|)$ \\
\hline Intercept & 4.59 & 0.0695 & 17 & 66.037 & .0001 \\
\hline Condition-MCI & -0.0019 & 0.0117 & 13070 & -0.162 & .871 \\
\hline Gender-Male & -0.1378 & 0.0122 & 13070 & -11.294 & .0001 \\
\hline Condition-MCI: Gender-M & 0.1061 & 0.0179 & 13070 & 5.934 & .0001 \\
\hline \hline
\end{tabular}


116 Ch. Themistocleous et al.

However, MCI and HC participants had great variation from the mean. And there was a clear difference between the two genders on vowel duration. To this purpose, we changed our initial model and added gender as a fixed factor and explored the interaction of gender and condition on vowel duration (adding only vowel as random intercept) (see Table 3). The findings in this case show a significant effect of the interaction of condition $\times$ gender on the $\log$-transformed duration.

\section{Discussion}

Overall, participants with MCI produce longer vowels than healthy controls. Longer vowels can be associated with an overall slower MCI speech than HC speech, which can be attributed to slower articulatory movements, greater cognitive processing and planning time of utterances. Nevertheless, future research is required to determine the durational properties of the speech of MCI vs. HC participants. Another important finding is that men MCI participants produce longer vowels than women MCI and HC participants. This finding may be attributed to gender specific effects on speech production in MCI Swedish speakers. Future research will investigate the effects of MCI vs. healthy controls on the duration of both vowels and consonants to establish if these findings are attested in consonants as well.

\section{Acknowledgements}

Riksbankens Jubileumsfond - The Swedish Foundation for Humanities \& Social Sciences, through the grant agreement no: NHS 14-1761:1.

\section{References}

Haley K.L., Overton H.B. 2001. Word length and vowel duration in apraxia of speech: The use of relative measures. Brain and Language 79, 397-406.

Fraser K., Lundholm Fors K., Eckerström M., Themistocleous C., Kokkinakis D., 2018. Improving the Sensitivity and Specificity of MCI Screening with Linguistic Information. LREC workshop: RaPID-2. Miyazaki, Japan.

Jarrold, W., Peintner, B., Wilkins, D., Vergryi, D., Richey, C., Gorno-Tempini, M.L., Ogar, J. 2014. Aided diagnosis of dementia type through computer-based analysis of spontaneous speech. In: CLPsych 2014, 27-36.

Themistocleous Ch. 2017. Modern Greek vowels and the nature of acoustic gradience. Phonetica 74, 157-172.

Themistocleous Ch., Kokkinakis D. 2018. THEMIS-SV. Proc. 4th European Stroke Organisation Conference. Gothenburg, Sweden.

Wallin A. et al. 2016. The Gothenburg MCI study: Design and distribution of Alzheimers disease and subcortical vascular disease diagnoses from baseline to 6-year follow-up. J Cer Blood Flow Metab. 36(1):114-131. 